\title{
LA CONFIABILIDAD EN LA ADMINISTRACIÓN EDUCATIVA
}

César S. Cortez Mondragón*

\section{LO QUE ENTENDEMOS POR CONFIABILIDAD EDUCATIVA}

\subsection{Conceptos Y OPINIONES}

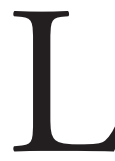

a confiabilidad viene de la confianza; es decir, de la seguridad que las personas tienen en la buena fe y en la opinión de un individuo o institución sobre aquello que se le ha encargado.

Profesor de Historia y Geografía, graduado en la Universidad Nacional Mayor de San Marcos. Diplomado en Educación en Población en la Pontificia Universidad católica del Perú. Maestría en Gestión y Administración Educativa en la Universidad Federico Villareal. Profesor y Director de diversas instituciones educativas, ex Director General de Educación Secundaria del Perú (1987-89). Fundador y Director del Instituto Buen Pastor (1993-1998). Dirigió la capacitación de docentes en Huaral 40 (Convenio UCSS-MINEDU) para las provincias de Huaral y Chancay (2007). Actualmente, se desempeña como catedrático de la Universidad Católica Sedes Sapientiae en las asignaturas de Historia de la Civilización, Pensamiento Educativo y Metodología de las Ciencias Sociales. Tiene publicados más de 10 libros de texto de educación secundaria, relacionados con geopolítica, psicología, economía, historia de la cultura peruana, historia universal y educación familiar. Ha hecho investigaciones sobre la historia de las escuelas y colegios parroquiales en el Perú y sobre la temática de los manuales de la asignatura de Historia del Perú para educación secundaria. Prepara un compendio titulado Historia del pensamiento educativo, que analizará el tema de la educación desde la prehistoria hasta el siglo xx. 
Por otro lado, la Educación está fundamentada en la axiología, en los valores. La ética y la confianza forman parte de la formación del desarrollo humano.

Autores como Manuel Kant, quien nos indica que «la educación tiene por fin dar al alma y al cuerpo toda la belleza y perfección que su naturaleza lleva consigo», o Enrique Pestalozzi, cuando dice que «la educación es el desarrollo natural y sistemático de la facultades del hombre» (Manrique: 2002), nos han expresado que la confiabilidad en los actos educativos es una parte importante de las actividades que se planifican para formar anímica y físicamente a las personas en desarrollo.

Esta caracterización que se realiza sobre la educación dentro de la axiología y de la confiabilidad, como valor esencial al hacer las cosas, nos conduce a decir que la enseñanza y el desarrollo de acciones educativas deben estar cimentados en un grado de confianza por parte del padre de familia, que entrega a su hijos para que los eduquen; del alumno, quien confiado recibe y se somete a la formación, y de las instituciones educativas, que deben establecer la parte más esencial de su labor en la confiabilidad. Así, la confiabilidad es un valor muy presente que el Estado debe hacer cumplir, pues se transforma en un derecho que se exige en el desarrollo de las tareas de todo sistema educativo.

Pocas veces, el presente tema ha sido tratado dentro de la administración educativa. Quizá se pensó que era parte del desempeño de la enseñanza y del aprendizaje; pero, ahora que mucho se habla de calidad educativa, resulta beneficioso establecer los conceptos, las características y las posibilidades para determinar lo que es la confiabilidad en la educación.

Por eso, primero se debe delimitar de qué confiabilidad se habla: de la confiabilidad de la enseñanza, del aprendizaje, de la seguridad o del aspecto económico. ¿De cual de ellas? En el presente estudio, se hará referencia a la confiabilidad que nace del trabajo que se realiza dentro de la administración 
educativa, que por cierto involucra métodos, personas, sistemas, valores, etc. Se trata de establecer que todas las instituciones educativas tienen el deber y la obligación de dar un grado de calidad en sus acciones y en sus actividades en general.

La confiabilidad se transforma en la carta de presentación que la institución educativa tiene ante los padres de familia y la sociedad. Sin duda, se convierte en la esencia y el valor de su trabajo cotidiano. Sin la confiabilidad, la institución educativa pierde su credibilidad y se transforma en una empresa sin valores éticos educativos. Por ello, los centros educativos planifican, realizan y procesan todas sus actividades, porque saben que de ese modo lo harán bien, con calidad y en beneficio del educando.

Entonces, la confiabilidad se podría señalar como la capacidad que tiene la institución educativa de poder realizar sus funciones o actividades con un grado de calidad y eficiencia. También se puede considerar como la gran probabilidad de realizar funciones previstas sin tener mayores incidentes o contratiempos bajo ciertas condiciones y en un tiempo determinado.

\subsection{ConfIABILIDAD, SEgURIDAD Y RIESGO EDUCATIVO}

El término confiabilidad se confunde con seguridad y riesgo cuando se refiere a fallas de los equipos, procesos o a la operabilidad de estos.

Mientras la confiabilidad se transforma en el valor total de las actividades educativas, la seguridad es el grado que determina la mayor o menor confiabilidad. A ello se agrega que cuando mayor seguridad existe, el riesgo disminuye y, por tanto, la confiabilidad se transforma en un medio de la calidad.

Entonces, se puede distinguir que la confiabilidad es el grado cualitativo; la seguridad, el cuantitativo; y el riesgo, el indicador para no cometer errores. 
En el siguiente cuadro, se especifica cada uno:

\begin{tabular}{|c|c|c|}
\hline Confiabilidad & Seguridad & Riesgo \\
\hline $\begin{array}{l}\text { Se nota en el grado de } \\
\text { trabajo que se realiza con un } \\
\text { buen funcionamiento. }\end{array}$ & $\begin{array}{l}\text { Se capta en la frecuencia } \\
\text { de tener menos daños } \\
\text { y equivocaciones que } \\
\text { enmendar. }\end{array}$ & $\begin{array}{l}\text { Está en la probabilidad de pérdidas y } \\
\text { dańos; en todo caso, en el número de } \\
\text { dańos personales o económicos, o en } \\
\text { el tiempo que se pierde. }\end{array}$ \\
\hline $\begin{array}{l}\text { Se observa en las personas, } \\
\text { equipos y en el sistema. } \\
\text { Se aprecia al captar } \\
\text { más ganancias y menos } \\
\text { pérdidas. }\end{array}$ & $\begin{array}{l}\text { Se aprecia porque no se } \\
\text { presentan daños en las } \\
\text { personas, equipos ni en } \\
\text { los sistemas. }\end{array}$ & $\begin{array}{l}\text { Se manifiesta en la frecuencia y en el } \\
\text { grado de pérdidas que puede tener el } \\
\text { equipo y el sistema. }\end{array}$ \\
\hline $\begin{array}{l}\text { Se puede valorar con el } \\
\text { grado de seguridad para no } \\
\text { contar con riesgo alguno. }\end{array}$ & $\begin{array}{l}\text { Se valora en la } \\
\text { concurrencia de los } \\
\text { riesgos, los peligros y en } \\
\text { las fallas. }\end{array}$ & $\begin{array}{l}\text { Se identifica los riesgos con la técnica } \\
\text { de HAzop. }\end{array}$ \\
\hline
\end{tabular}

Para llegar a la confiabilidad, se tiene que observar que la institución educativa haya llegado a un grado de análisis; es decir, que pueda pasar el examen de eficiencia y que sus fallas sean mínimas, entendiéndose por fallas las debilidades y desaciertos frecuentes al efectuar cualquier labor educativa. Las fallas determinan el grado de confiabilidad, competencia y credibilidad. En efecto, la confiabilidad se precisa en el manejo y dominio de todas las fallas que acumula la institución educativa.

En principio, la relación directa entre fallas y confiabilidad se establece cuando se descubre que:

—Existe una experiencia óptima al realizar una determinada labor.

- Se tiene personal de calidad con el que se cuenta en cualquier momento. 
—Existe dominio en el manejo de instrumentos que facilitan el cumplimiento de la actividad.

- Se hace el trabajo en un tiempo record, con bajos costos y menor presencia de errores.

Regularmente, se establece que existe confiabilidad cuando los errores son menores o, con frecuencia, nulos. Las fallas deben estar reducidas a características técnicas manejables; así quien realiza el servicio, podría seguir trabajando sin afectar el producto final.

Los análisis administrativos pueden establecer dos clases de fallas:

\section{Falla funcional}

Es aquella falla que está prevista o esperada. El ejemplo característico es el de los tiempos de aprendizaje: algunos padres de familia creen que su hijo debe aprender a leer a los tres o cuatro años; por ello, obligan los profesores a agilizar la enseñanza para su posterior aprendizaje y no tienen presente el desarrollo mental. Otro caso se produce cuando no se sabe distinguir entre evaluación continua y evaluación programada; por eso, se tiene muchos desaprobados en la escuela. En general, ocurre cuando estamos convencidos de que la planificación no es parte de la administración educativa y se realiza improvisaciones muy frecuentes.

\section{Falla potencial}

Es aquella que está por encima de lo planificado. Sus ajustes se realizan gracias a las pruebas de funcionamiento, que buscan identificar problemas para solucionarlos. Por ejemplo, cuando se desarrolla una epidemia o enfermedad entre los educandos y estos faltan al colegio; en consecuencia, 
se tiene que hacer correcciones en la programación temática. Otro ejemplo se produce cuando el cambio de la estructura curricular no prevista — sobre todo legal— condiciona el bajo rendimiento escolar.

\subsection{LO QUE INVOLUCRA LA CONFIABILIDAD EDUCATIVA}

Los críticos del proceso administrativo siempre determinan que la confiabilidad se encuentra en los planificadores o en aquellos que hacen la gestión educativa; es decir, los que son operativos o que ejecutan las labores educativas. Este error ha llevado siempre a buscar culpables antes que buscar soluciones. En otros casos, se coloca soluciones-parches que ayudan solo a poder aliviar lo que debería transformarse en manejo de experiencias sobre el análisis de hechos.

Carranza Díaz (2006) indica que existe confiabilidad cuando están presentes tres términos claves:

a) La consistencia estable: Esto quiere decir que los resultados óptimos no deben variar, aun cuando sean dados en diferentes tiempos. Cuando algo es consistente, el éxito es continuo. La calidad se maneja como algo cotidiano y los errores son tan manejables que, dentro del trabajo cotidiano, no se notan o forman parte del quehacer controlable, por no decir que no existen.

a) Lo predecible: Se sabe que algo tiene que producirse y, por eso, se espera con mayor seguridad y conocimiento. Esto quiere decir que el trabajo educativo se maneja con certeza; además, se conoce que los pasos se darán con la mayor precisión y calidad posible. Por ejemplo, si se observa que los métodos son adecuados al realizar 
la acción educativa, los instrumentos y las estrategias también son convenientes, e incluso las personas se sienten contentas con los eficientes resultados. Sin embargo, en el aspecto educativo, se debe manejar lo impredicible; es decir, aquello que se puede presentar como recurso de último momento ante lo inesperado. Esta caracterización, si ocurre por la precisión de lo planificado, está incluida; el buen administrador educativo debe tenerla como una opción. En otros casos, es necesario ser prevenido y tener otra «carta bajo la manga».

b) La claridad de metas y objetivo: Cuando se sabe lo que se puede y se tiene claro lo que se quiere, entonces se puede decir que se conoce la realidad y que se la puede manejar para beneficio de nuestros fines. Sin duda, se conseguirán los resultados esperados. Tener siempre claridad en los resultados propicia el mejor logro de metas (cantidad) y objetivos (calidad). Es más, con la claridad se obtendrá, en forma precisa y eficaz, lo que se quiere.

Estas mismas características son mencionadas por Neil Salkind (1999) y Hernández Sampieri (1999). Ellos concluyen que la confiabilidad funcionará en los tiempos más variados, con la misma capacidad de realización y con los mismos resultados.

La confiabilidad operacional (que es el manejo o la ejecución de tareas) es siempre a la que se hace referencia para observer las fallas y determinar la seguridad del trabajo. Ciertamente, las fallas tienen elementos significativos en donde se encuentra mucho de lo que trata la confiabilidad. Como se ha mencionado en líneas anteriores, estos elementos esclarecen las debilidades y motivan a buscar o a encontrar los errores. Esos elementos significativos pueden ser los siguientes: 
—Las pérdidas constantes.

—Las reparaciones frecuentes.

-Los accidentes previsibles.

-El descontento, tanto del usuario como de los directivos.

- La baja calidad que se ofrece y que comprueba el usuario.

—El menor rendimiento en las diferentes áreas educativas.

—La pérdida de eficiencia, que determina la baja calidad.

—Los problemas del ambiente (algunos posibles de solucionar).

—Los resultados negativos, que denotan debilidades visibles.

—El mayor consumo de energía, cuyo desperdicio hace perder calidad y eficiencia a la empresa.

- La pésima comunicación o mal entendimiento entre las personas de la institución. Esta produce demoras y una pésima imagen institucional, y después degenera en el clásico caos administrativo.

—La falta de adiestramiento, causada por la falta de planificación.

Como se puede observar, el temario puede ser largo y lleva a especular la presencia de puntos estratégicos. Estos puntos, en un primer momento, pueden dejarse pasar; pero después se transforman en problemas mayúsculos. La razón, que parece ser sencilla, se complica porque se olvidan cosas cotidianas. 
1.3.1. Las directivas no son eternas y pocas veces se nota que presentan fallas en el trabajo gerencial

Si se observan constantemente desaciertos y los usuarios (padres de familia y educandos) se mortifican o aparentan soportar las debilidades de las autoridades, significa que existe falta de renovación (preparación constante), cambio de paradigmas (contar con gente que no acepta otras alternativas o cambios) o soluciones propuestas a destiempo.

1.3.2. Los sistemas, por más perfectos que aparenten ser, necesitan revisión constante para ver su ejecución y mantenimiento

Es necesario observar si un sistema puede ser obsoleto, porque ya no responde a las expectativas; es decir, cuando se aprecia que algo ya cumplió su misión, se necesita cambiarlo. Los sistemas cambian con el desarrollo de las nuevas inquietudes o expectativas; sin embargo, se cree que aquello que se hizo bien siempre se hará bien. Cuando, sin darse cuenta, se persiste en los errores, otros están percibiendo las fallas y debilidades.

1.3.3. Los medios de comunicación no responden al fluido intercambio de ideas para que todos los elementos del sistema respondan con las mismas expectativas

Cuando se transforman los elementos en voluntad de sordos, se produce la incomprensión de terminologías, de símbolos de comunicación, y la variación de paradigmas y mentalidades. Cuando la falta de comunicación 
es intencional, o producida por incomprensiones entre los miembros de una comunidad, la confiabilidad está siempre condicionada; mientras que los problemas se derivan de este sistema de comunicación. Se ha podido observar la destrucción de sistemas prósperos por la falta de comprensión en los símbolos de comunicación.

\subsubsection{Al sentir que se está frente a una subida de costos que no se puede manejar}

Los cambios económicos afectan presupuestos. En algunos casos, se ha llegado a tener pagos obligatorios sin motivo y pérdidas de ganancias esperadas. Los cambios en precios, ganancias e inversiones, así como todo lo relacionado con la sustentación de lo económico, primero produce desconcierto; luego, desazón, y, finalmente, termina examinando todo lo planificado, lo previsto y la capacidad de acierto.

\subsubsection{Notables y frecuentes deficiencias en el personal y en los asesores}

Si los problemas siguen persistiendo con las mismas características y tiempos, o los asesores no buscan alternativas de solución e insisten con las antiguas por serles cómodas, el personal, sin darse cuenta, ha caído en la monotonía, que es el inicio de lo deficiente. Al ver que ya los errores no son manejables, cuando luego agobia las debilidades con continuas deficiencias, entonces se siente que las deficiencias se mantienen como parte de las soluciones. En fin, se vuelve a caer en los mismos arreglos, los mismos análisis y los mismos criterios. Así, se puede convertir en repetidor cuando se está cayendo en la 
monotonía confiable; por ello, la costumbre hace familiar las soluciones y la objetividad puede estar comprometida. Aquí es necesario buscar objetivos, metas, visión y misión, los que dieron el progreso a la empresa y al sistema. Estas pueden ser las formas-guía de cómo las personas quieren manejar lo que se está haciendo.

\subsubsection{La modificación de valores de la visión institucional}

Se trata de desviar lo que identifica a la institución educativa, ya sea por un afán de ganancia o modernidad, proponiendo algunas alternativas que desdicen el trabajo de calidad o la razón por la cual la institución ampara su confiabilidad. La idoneidad es parte de la confiabilidad; cuando este $\mathrm{u}$ otros valores sufren cambios, ya sea por influencia de intereses o por formas bruscas de resolver lo no previsto, se hace que se olvide la esencia de lo propuesto. Cuando no están claros los recursos ideológicos desde el inicio del trabajo, casi siempre, en un momento de debilidad, llegan a imponerse valores en forma sesgada o a suprimirse porque molestan a la consecución de los nuevos fines.

Estas puntualizaciones significativas son motivo para pensar que la confiabilidad involucra a todos; es decir, que es un don que la institución maneja como suyo. Cuando la confiabilidad educativa se transforma en un trabajo operativo, hasta cotidiano se transforma en un hábito que se cumple con mucha eficiencia. La dirección, el sistema, los métodos y el equipo ejecutante trabajan bajo una armonía. Los administradores y los economistas la llaman armonía del desarrollo; los educadores, calidad educativa. 


\subsection{LOS FACTORES DE LA CONFIABILIDAD}

En la práctica, la confiabilidad requiere establecer ciertos factores universales, todos ellos existentes dentro de la realización del servicio educativo. Así tenemos los siguientes:

\subsubsection{Confiabilidad en el equipo que realiza el servicio}

Se sabe que las instituciones están más allá de la infraestructura, que es lo que causa impresiones a padres de familia, alumnos y profesores; también que es más que los sistemas que se aplican. Lo fundamental consiste en la dirección y gestión institucional, la participación de los padres de familia y en los edcandos, elemento centro de todas las operaciones educativas. La confiabilidad consiste en obtener el entrenamiento, los valores personales de los miembros, los conocimientos que tiene la comunidad educativa (servicio, profesores y directivos) de la operación que se realiza, y hasta la confianza y responsabilidad. Así, se puede determinar el valor de la evaluación y la demostración de la acreditación.

\subsubsection{Confiabilidad en el medio ambiente en el cual se opera}

El medio ambiente es todo lo que rodea a las actividades educativas. En las escuelas y colegios, el ambiente favorece la confiabilidad y la hace propicia, tanto que es lo más impresionante para todo aquel que busca calidad educativa. Corresponde al medio ambiente: 
—La estructura apropiada de los locales.

—Los materiales educativos que se usan para realizar una ejecución.

-El medio social como componente comunitario.

—La Autoestima y la fe de los usuarios.

\subsubsection{La carga de trabajo que debe ejecutarse}

Mejor sería denominada como cantidad o monto por hacer. Aquí, se tiene que ver la capacidad del proyecto que se desea realizar, los procesos o etapas, y la singularidad de lo que se quiere conseguir. La capacidad de hacer las cosas y la increíble manera de saber que se hará bien facilitan que la obra sea posible de realizar; para ello, es necesario que se tenga capacidad para terminar y que se considere al capacitado para hacerlo de manera creativa y diferente o especial.

\subsubsection{Las probabilidades de falla}

Son establecidas por imprevistos o por la naturaleza humana del servicio, el control manejable y la corrección para cumplir los objetivos y metas. Sin tener miedo al error, muchas veces se debe llegar a acumular experiencias; tantas que las equivocaciones se colocan como parte de la planificación y del proceso (margen de error). 


\subsubsection{La capacidad de valores fundamentales}

Por una acción educativa y formativa, en la entereza de buscar la vocación del servicio, la grandeza está en la singularidad de hacer las cosas porque las hacemos bien. Las personas, junto a las instituciones, se identifican por sus acciones o actitudes. Tal puede ser el juicio de valor que la identificación crea el margen de la fama, etiqueta de identificación, el logotipo que vende y hasta el prejuicio de siempre hacerlo bien.

\subsubsection{El uso de las mediciones y las pruebas de funcionamiento}

Se trata del control en el inicio, en el proceso y en el final. Es también el producto final (el trabajo realizado, la obra terminada) de un control constante, así nadie duda de la eficiencia. Muchas instituciones educativas basan su tarea en demostrar que los errores no cuentan en lo que hacen, porque todo esta y estuvo siempre bajo su control. Por ello, se trata, desde los inicios, de llegar a tener la seguridad de que no habrá desviaciones; así, lo modificable está bajo control y los errores son experiencias necesarias para que se tenga fe en lo que se hace o en lo que se ha propuesto realizar.

\subsection{LA CONFIABILIDAD Y LA CALIDAD EDUCATIVA}

Mucho se ha escrito sobre la "calidad educativa». Aunque no es propósito, en este texto, extender una explicación sobre ella, no se puede dejar de relacionarla con la confiabilidad, puesto que estuvo ya estructurada desde el momento en que Taylor estableció sus procesos, Sherwhart hizo sus esbozos, 
Deming explicó sus modificaciones y hasta Ishikawa estableció sus pasos. En todos ellos, se encuentra la explicación de hacer una buena administración, la que hoy se ha aplicado al trabajo educativo y que permite la mejora con mucho profesionalismo.

A partir del estudio de estos grandes pensadores, se debe rescatar que la «tecnología educativa», en su momento, pretendió realizar cambios y mejoras, aunque de manera muy cognitiva. Tal es el caso que para llegar a una mínima confiabilidad, se tiene que realizar un proceso simple de trabajo administrativo (cf. Sexto 2002) como es el PHVA. ¿Qué significa esto? Pues, planificar, hacer, verificar, actuar.

A continuación, se realizará la explicación de cada uno de ellos:

a) Planificar: Es la etapa por la cual se establece objetivos y procesos para conseguir resultados de acuerdo a ciertos valores que se quiere alcanzar. Es necesario recordar que la planificación educativa no pretende establecer el producto acabado, porque se trabaja con personas. La planificación da la seguridad de saber lo que se hace y de la mejor manera; ya sea a corto o largo plazo, la planificación establece la realización ideal del trabajo.

b) Hacer: Consiste en realizar o implementar los proceso o actividades. En educación, representa uno de los principios de Delors, como parte de la «calidad educativa». Todo el aprendizaje se confirma en hacer, manejar o manipular; es decir, en realizar lo planificado. El hacer, en el campo educativo, es la confirmación de lo aprendido o, por lo menos, la comprobación de que se ha entendido bien las cosas.

c) Verificar: Es la necesidad de realizar el seguimiento, la medición de los procesos y el análisis de las respuestas. La tecnología educativa ha implementado muy bien esta parte del trabajo. El hacer educativo 
es parte de la comprobación de lo enseñado; en lo administrativo, es la verificación de lo planificado. Muchas de estas formas de verificar se confirman en la observación de la repetición, como forma de aprendizaje, y en la confirmación objetiva del trabajo administrativo.

d) Actuar: Este paso complementa al hacer, pero tomando las experiencias del informe de resultados. Es ir corrigiendo $y$ mejorando procesos. En cierta manera, se vuelve a invertir o planificar de nuevo. Es la realización de las ideas, pero mejoradas. La actuación es la confirmación del hacer: «cuando sentimos que ya lo hemos hecho bien, verificamos». Es verificar y asegurar que es confiable. Una baja mínima en la estructuración del trabajo educativo puede conducir a la caída de la calidad y a la caída de la confiabilidad. Se podría decir que la metodología PHVA da lugar a la confianza para hacer bien los procesos humanos y los procesos materiales en educación.

En estos pasos, hay etapas que pueden interpretarse como preventivas; por ejemplo, las observaciones para los pasos 1, 2 y 3 , porque tratan de establecer la ejecución con éxito. Mientras que la ejecución del paso N. ${ }^{\circ} 4$ es para determinar las correcciones, pues ayuda a detectar deficiencias para acciones preventivas posteriores. En el sistema educativo, la confiabilidad exige, tal vez, un N. ${ }^{\circ} 5$ por la razón de no tener el producto completamente acabado.

e) El ciclo de mejora continúa: Significa seguir trabajando en la formación del educando, porque la confiabilidad se basa en una búsqueda incesante. Por estar contenida en cada una de las etapas y a la vez sintetizar a todas, se caracteriza por un control continuo y por la realización de los procesos a través de la búsqueda de la 
calidad educativa. En esta etapa, interviene la verificación y la actuación.

\section{ACTUAR}

(Acciones

\section{conectivas)}

3. VERIFICAR

(Acciones

Eliminar

Problemas

Detectados

evaluativas)

1. PLANIFICAR (Acciones preventivas) 


\section{METODOS Y FORMAS MÁS USADAS PARA DETERMINAR LA CONFIABILIDAD}

La confiabilidad siempre está unida a la seguridad. Muchas personas cuando hacen algo confían en que lo harán bien, porque tienen la seguridad de sus métodos y formas para hacerlo; pero cuando se trata de delegar a otras personas la realización del trabajo, la confiabilidad disminuye y se necesita sugerir ciertos métodos con características especiales que ayudan a mejorar la realización de las labores.

En el sistema educativo, la confiabilidad en la realización de tareas se condiciona por la falta de planificación, el uso acostumbrado de métodos arcaicos y la imposibilidad de renovación, o también por la falta de recursos, desde personales hasta económicos. En este estudio, se desea exponer los métodos que la administración educativa ofrece, a la espera de que la confiabilidad siempre determine el mejor grado para hacer las cosas y los trabajos con creatividad y eficiencia.

A continuación, se ofrecen algunos de estos métodos, estableciendo sus características generales, las ventajas que ofrecen y las posibles desventajas a las que se puede estar condicionado:

\begin{tabular}{|l|l|l|l|}
\hline \multicolumn{1}{|c|}{ Método } & \multicolumn{1}{|c|}{ Características } & \multicolumn{1}{c|}{ Ventajas } & \multicolumn{1}{c|}{ Desventajas } \\
\hline $\begin{array}{l}\text { Análisis preliminar } \\
\text { de riesgos }\end{array}$ & $\begin{array}{l}\text { Define riesgos y sistemas } \\
\text { que ayudan a conocer } \\
\text { mediante una simple } \\
\text { observación o cateo. }\end{array}$ & $\begin{array}{l}\text { Como lluvia de } \\
\text { ideas, es muy útil } \\
\text { en una primera } \\
\text { etapa. }\end{array}$ & $\begin{array}{l}\text { Tiene alcance limitado. } \\
\text { Consiste en lo que } \\
\text { siempre se hace para } \\
\text { evitar toda clase de } \\
\text { errores. }\end{array}$ \\
\hline $\begin{array}{l}\text { Análisis de modos } \\
\text { de falla y efectos }\end{array}$ & $\begin{array}{l}\text { Analiza todos los } \\
\text { modos de fallas de los } \\
\text { componentes, sean } \\
\text { simples o complejos. }\end{array}$ & $\begin{array}{l}\text { Es muy parecido al } \\
\text { anterior. Es fácil. Se } \\
\text { acepta; pero no se } \\
\text { estandariza, no es } \\
\text { matemático. }\end{array}$ & $\begin{array}{l}\text { Examina fallas no } \\
\text { peligrosas; su uso es } \\
\text { común. }\end{array}$ \\
\hline
\end{tabular}




\section{LA CONFIABILIDAD EN LA ADMINISTRACION EDUCATIVA}

\begin{tabular}{|c|c|c|c|}
\hline $\begin{array}{l}\text { Análisis de puntos } \\
\text { críticos }\end{array}$ & $\begin{array}{l}\text { Investiga el ranking de } \\
\text { los componentes para } \\
\text { actualizar sistemas. }\end{array}$ & $\begin{array}{l}\text { Técnica } \\
\text { estandarizada } \\
\text { fácil de aplicar y } \\
\text { entender. }\end{array}$ & $\begin{array}{l}\text { ¡Cuidado! A veces no } \\
\text { ve factores humanos, } \\
\text { pero si causas de fallas } \\
\text { comunes o interacción } \\
\text { de sistemas. }\end{array}$ \\
\hline $\begin{array}{l}\text { Análisis del árbol } \\
\text { de fallas }\end{array}$ & $\begin{array}{l}\text { Sirve para graficar. } \\
\text { Comienza con un } \\
\text { evento y busca la } \\
\text { combinación de fallas } \\
\text { que lo causan. }\end{array}$ & $\begin{array}{l}\text { Es una técnica muy } \\
\text { aceptada. Es una } \\
\text { buena forma para } \\
\text { encontrar relación } \\
\text { entre las fallas. }\end{array}$ & $\begin{array}{l}\text { Es difícil de entender. } \\
\text { Tiene que ser } \\
\text { explicada por quien } \\
\text { lo hace, puesto que } \\
\text { le da un tratamiento } \\
\text { matemático-lógico } \\
\text { complejo. }\end{array}$ \\
\hline $\begin{array}{l}\text { Análisis de } \\
\text { eventos }\end{array}$ & $\begin{array}{l}\text { Comienza con eventos } \\
\text { principales y se } \\
\text { examinan secuencias de } \\
\text { eventos alternativos. }\end{array}$ & $\begin{array}{l}\text { Puede verse } \\
\text { secuencias, seguir } \\
\text { procesos y localizar } \\
\text { fallas para poner } \\
\text { alternativas. }\end{array}$ & $\begin{array}{l}\text { No sirve para cambiar } \\
\text { las secuencias, tampoco } \\
\text { para quedar en el } \\
\text { análisis de los detalles. }\end{array}$ \\
\hline $\begin{array}{l}\text { Análisis de riesgos } \\
\text { y operabilidad } \\
\text { (HAZOP) }\end{array}$ & $\begin{array}{l}\text { Incluye causas y efectos. } \\
\text { Son los cambios en las } \\
\text { variables de una escuela. }\end{array}$ & $\begin{array}{l}\text { Adecuado para } \\
\text { grandes escuelas } \\
\text { y para detallar } \\
\text { eventos. }\end{array}$ & $\begin{array}{l}\text { Involucra un grupo } \\
\text { personas. Es tedioso } \\
\text { para evaluar si } \\
\text { falta costumbre o } \\
\text { preparación. }\end{array}$ \\
\hline $\begin{array}{l}\text { Análisis de causas } \\
\text { y consecuencias }\end{array}$ & $\begin{array}{l}\text { Comienza con eventos } \\
\text { críticos y trabaja hacia } \\
\text { adelante usando el árbol } \\
\text { de evaluación. También } \\
\text { analiza el pasado e, } \\
\text { inclusive, las fallas. }\end{array}$ & $\begin{array}{l}\text { Hay flexibilidad } \\
\text { bien documentada. } \\
\text { La secuencia es } \\
\text { clara. Puede servir } \\
\text { para la planificación } \\
\text { estratégica de largo } \\
\text { alcance. }\end{array}$ & $\begin{array}{l}\text { Posee diagramas } \\
\text { muy grandes para } \\
\text { ser interpredos con } \\
\text { facilidad. Es una } \\
\text { desventaja para el uso } \\
\text { concreto de árboles de } \\
\text { fallas. }\end{array}$ \\
\hline
\end{tabular}

Aunque el cuadro no permita detallar el uso dentro del campo educativo de las escuelas y colegios, porque muchas de estas metodologías se usan de manera particular, se quiere, en las siguientes páginas, extender algunas por estar relacionadas con la administración en general. 


\subsection{LA ESTADÍSTICA Y LA CONFIABILIDAD}

La estadística es una ciencia que, sirviéndose de las matemáticas y del cálculo fundamentado, analiza e interpreta la información a fin de poder hacer previsiones sobre un comportamiento futuro.

Los métodos estadísticos son muy utilizados en las ciencias sociales. En educación, son una garantía de la confiabilidad. El análisis estadístico es determinante para fijar fenómenos casuales y dudas, y para valorar y eliminar irregularidades.

El uso más frecuente esta en la «estadística descriptiva»; es decir, cuando los datos recogidos sirven para graficar y poner de manifiesto las posibles relaciones de dependencia entre los datos y la medida que permite el estudio que se está realizando. Entre los ejemplos más característicos encontramos a la población escolar, y al tamaño o mensura de elementos para planificar aulas, laboratorios, tiempos, etc. El otro uso es el de la «estadística inductiva». Se trata del estudio con muestras, cuyo cálculo de probabilidad es utilizado como un conocimiento para deducir con certeza. Aquí están los valores centrales, las desviaciones, el cálculo de los errores, entre otros. (Planeta 2001).

Sin embargo, la estadística, dentro del grado de confiabilidad, es criticada desde que se acepta que puede demostrarse con los siguientes elementos:

\section{—Datos falsos}

—Análisis incorrectos

—Manipulación de los resultados

En otros casos, el sistema aplicado no ha sido revisado y se llega a pecar en la confiabilidad por los siguientes motivos: 
- Ignorancia en el manejo de los datos

—Desidia de quien maneja la estadística

-Mala interrelación o poca intuición en el manejo de los resultados

A ello se debe agregar que la seguridad mayor se le otorga cuando se cree que los sistemas de computación y la seguridad de los softwares son infalibles, sin comprender el manejo y la disposición de quien los realiza. Por eso, el físico Kapitsa indica que uno de los tres tipos de mentira es la estadística (los otros tipos son la mentira piadosa y la mentira desvergonzada).

Entonces, ¿cómo utilizar a la estadística dentro de la confiabilidad? Del siguiente modo:

- Primero, debe usarse dentro de un índice de probabilidades, pero no como el único dentro de la confiabilidad.

- Se debe establecer su análisis desde el uso y concepción como término de probabilidad.

- No debe ser empleada cuando hay predicción de fallas con datos cuestionables.

-En la estadística descriptiva se usa para confirmar el dato; en la estadística inductiva, como indicador de valor en el promedio y en la tendencia central.

Por eso, el uso de la estadística en educación más tiene que ver con el operador antes que con el estadístico. En fin, su uso es un elemento de control y mejoramiento de la calidad, pero con el apoyo de otros elementos que caracterizan la confiabilidad. 


\subsection{ANÁlisis DE CRITICIDAd O DE LOS PUNTOS CRíticos}

Los puntos críticos son las debilidades; Estas deben ser tomadas en cuenta el administrador educativo. Algunos puntos críticos son inherentes a la estructura del sistema y tienen que manejarse como fallas; otros surgen como parte del proceso. En ambos casos, se busca controlarlos.

Todo punto crítico se puede situar en:

- La frecuencia de una falla.

- El impacto operacional o el porcentaje que produce la falla.

- El nivel con que, habiéndose producido la mejora, vuelve a repetirse la falla.

- El tiempo con el que se nota la deficiencia.

- El costo o gasto de la reparación o el mejoramiento donde se encuentra el punto crítico.

- El impacto ambiental sobre el cual influye el punto crítico.

- En educación, también se debe tomar en cuenta las consecuencias humanas predecibles.

Por eso, es importante determinar (diseñar) las áreas en las cuales se encuentran los puntos críticos. Cuando se planifica, se sabe, y muchas veces se siente, si son altas, medias o bajas. Es allí cuando los directivos de la educación buscan las estrategias de solución. En otros casos, se comienza a jerarquizar el impacto del daño, teniendo en cuenta el aspecto global como consecuencia, con el fin de facilitar las decisiones. Si embargo, también se pueden realizar: 
—Definiendo los alcances y propósitos para el análisis.

—Estableciendo criterios de evaluación.

—Anotando el grado de confiabilidad.

Los administradores, cuando identifican con claridad los puntos críticos, se proponen lo siguiente:

- Se fijan las prioridades dentro del sistema para categorizar las soluciones en el aspecto global; por ejemplo, los conocimientos básicos de los alumnos, la preparación de los profesores según su especialidad, la calidad educativa del padre de familia, la infraestructura con que contamos, la organización, etc.

- Se observa la forma cómo se administra los recursos que solucionarán los problemas que surjan en los puntos críticos; por ejemplo, si existe facilidad para obtener recursos, mucha o poca burocracia en los trámites, gastos operativos, previsión de efectivos, etc.

- Se determina el impacto en el negocio: la ganancia y la inversión; por ejemplo, los costos, las ganancias, la capacidad contable, el financiamiento factible, los pagos adecuados y controlados, la verificación de gastos, los controles económicos, etc.

- Se aplican metodologías de confiabilidad; aquellas que están dispuestas en el conocimiento de los que forman la empresa educativa. Para ello, se necesita todo un plan o sistema rápido que aplicar, dentro del cual todos se sintiesen seguros. Se sabe que todas las instituciones educativas tienen sus sistemas de confiabilidad; por muy pequeña que sea o no la conozca con ese nombre, saben en qué momento esto o aquello debe y está controlado para que salgan bien las cosas. 
Para ello son necesarios los índices de información, con los cuales se puede establecer si el punto crítico es confiable en el manejo o difícil; también se necesita una mayor atención. Por ejemplo, se acude al índice de la estadística, al establecimiento de rangos, a la frecuencia de fallas, entre otros. Toda esta información tiene que ser manejada por los participantes del trabajo según su responsabilidad, porque allí radica que se mantenga o desaparezca el punto crítico.

Cuando ya se sabe cuáles son los puntos críticos y se pueda establecer su manejo o solución, entonces viene la precisión de la confiabilidad educativa, que se dividiría en:

—Confiabilidad humana

—Confiabilidad del proceso

—Confiabilidad en el mantenimiento del sistema

-Confiabilidad operacional

Para saber si los puntos críticos están completamente evaluados, es necesario, para un próximo trabajo educativo, tener en cuenta lo siguiente:

- Tablas de estándares, muy usados en rendimiento escolar.

- Sistema de computación con un software confiable, que ayude a la rapidez de las soluciones.

- Implementación de las áreas donde se dan los puntos críticos; por ejemplo, si es en personal, materiales usados, inspección y dirección, etc. 


\subsection{ANÁLISIS DE LOS RIESGOS OPERATIVOS: HOZAP}

El Hozap es una técnica para identificar riesgos y problemas, que impide una operación deficiente. Según su forma sistemática, reduce la posibilidad de que algo pase sin ser analizado. Se considera como parte de la seguridad del proceso para la protección personal, institucional y comunitaria. Tiene dos objetivos:

1. Identificar riesgos; es decir, identificar características de planta, sistemas, equipos y procedimientos que puedan presentar accidentes.

2. Identificar problemas de operatividad que puedan ser producto de fallas de diseño y operatividad. Todos ellos contribuyen a desmejorar o dañar, con sus fallas y riesgos, un trabajo tan bien preparado.

Además, se hace operativo con los siguientes elementos:

a) Los participantes: Son los que tienen que ver con el problema (los dirigentes o los trabajadores). El desempeño de los participantes afecta al éxito o al fracaso de lo que se ha propuesto o planificado. Se toma un número pequeño de participantes; por ejemplo, un promedio de dos o tres y un máximo de diez personas. Con ellos se establece consultas, porque conocen detalles de su sector, y se realiza desde una conversación hasta la estructura de una operación de análisis de riesgo. Lo importante es saber que quienes realizan el trabajo tienen la certeza de que los riesgos operativos son manejables.

b) La duración: Es el tiempo que corresponde a ocho horas teóricas en las que se introduce el sistema antes del riesgo. Se hace para que se tome conocimiento del trabajo y, a la vez, se analicen los resultados. 
Es recomendable procurar no tensarse con un tiempo mayor, porque disminuye la vitalidad del trabajo. Mucho tiene que ver la relación del trabajo con el tiempo que demora realizarlo. No se necesita que el cansancio malogre la calidad y haga perder la confiabilidad.

c) El contenido: Consiste en conocer los objetivos, la conformación del equipo, la duración requerida, la aplicación dentro de los proyectos, las instalaciones existentes, los procesos continuos; es decir, todo cuanto se hará o se podría hacer. Los contenidos deben ser claros y precisos. Las órdenes difusas, establecidas con dudas, favorecen la equivocación.

d) Los resultados: Es la documentación que servirá para la aplicación de una nueva forma de hacer las cosas y bien. La efectividad debe ser la meta. Los resultados para la eficacia deben ser previsibles. Lo que se necesita comprobar es que tengan un grado óptimo y satisfaga a todos, solo así se establece el adelanto de las ciencias y del conocimiento.

e) La documentación: Son los diferentes escritos, informes orales, entre otros, que ayudan a esclarecer o a mejorar el trabajo y la confiabilidad. Aquí se recogen aquellas opiniones y documentos relacionados, desde los favorables hasta los absurdos, para lograr el convencimiento sobre la confiabilidad. En este caso, el orden de los planos, de las hojas de ruta, del plan, de la hoja de actividades y de los agregados a la documentación oficial son necesarios para que cuando se necesite su consulta, se conozca su ubicación, además de quién los tiene y para qué sirve.

f) Los recursos: Son todos aquellos que se necesitan para realizar la técnica. Esta técnica, en educación, se puede usar como parte del monitoreo de operaciones; de esta manera, se puede favorecer al desempeño de las actividades planificadas. 


\subsection{HozAP EDUCATIVO}

Debe utilizarse como documento en la planificación de la actividad educativa detallada, que indique la guía sin equivocaciones. Se entiende que los profesionales que van a realizar el trabajo son expertos que tienen un conocimiento acumulado, una experiencia, y que conocen todo el proceso del trabajo; o bien que son personas con quienes se cuenta, por su imaginación, para hacer uso de los recursos e instrumentos en cualquier momento que se produzca una falla o una equivocación. En algunos casos, adelantarse a la falla es importante si la situación o la intuición lo permiten.

Se sabe, debido a que todo está estudiado, comprobado y perfectamente adecuado, que la labor se ha planificado porque las personas encargadas del caso conocen, con una descripción detallada, todo el proceso del proyecto o del trabajo que se debe realizar. Además, se establecen las posibles desviaciones y se proponen cambios o se previenen debilidades, de modo que se puedan controlar las fallas y los riesgos. Asimismo, a medida que va viendo el desarrollo del proceso o de la actividad educativa, se van revisando la intención y el propósito de cada etapa. Cuando se ha identificado la falla o desviación, inmediatamente los profesionales hacen suya la operación de riesgo y solucionan la problemática. Luego, analizan las consecuencias para evitar otros riesgos y problemas operativos.

En la existencia de la desviación del propósito o cambio posible, debe tenerse en cuenta lo siguiente:

a) Si el proceso también puede convenir y mejorar durante su realización, en tal caso el apoyo mantiene la desviación, hasta que después se analicen sus consecuencias.

b) Se ve si hay falta de voluntad para hacer o remedir la falla en algunos elementos que hacen la operación. 
c) El costo calculado (económico, de tiempo, de personas y de valores) responde a lo esperado o planificado.

d) Se debe tener en cuenta que en todo momento es posible la pérdida de la credibilidad y la calidad ganada.

En cuanto a la documentación, se debe tener en cuenta la necesidad de conocer lo planificado y si se tiene todos los pasos de la planificación, el desarrollo y la evaluación; en consecuencia, lo planificado debe salir conforme está en el papel o en el proyecto. Para ello, se debe tener en cuenta las siguientes recomendaciones:

a) El análisis de informes para enterarse de lo que ha pasado en otras secciones o para tener la visión de otros elementos del grupo.

b) La apreciación de personas que estuvieron fuera del evento; las observaciones de estas tienen validez (medio de opinión masiva).

c) La opinión de los involucrados, cuyo análisis puede hacer mejor futuras actuaciones.

d) La comparación con los documentos oficiales y los que se manejan en el desarrollo, de modo que ayuden a ver otras opiniones.

Un trabajo educativo, como los eventos que cotidianamente se llevan acabo en las instituciones educativas (por ejemplo, según el calendario cívico o protocolar), se desarrolla con una planificación casi monótona; en otros casos, tradicionales. Sin embargo, esta forma de trabajo puede conducir a un mejoramiento de los mismos y a la búsqueda de hacer las cosas con mucha confiabilidad. 


\subsection{LA ORGANIZACIÓN IMPLEMENTADA PARA EL CLIENTE (CRM)}

Esta vez se ha tomado la referencia de la administración de empresas. La referencia es la traducción de Customer Relationship Management (CRM) (cf. López: 2004), cuyo significado es el manejo de la implementación y organización empresarial para la retención del cliente. Esto es hacer posible que el cliente se encuentre satisfecho por la venta de nuestro producto o por el servicio que le prestamos y, por lo tanto, de nuestro trabajo. Posiblemente, esto se manifieste en el incremento de las ganancias.

Cuando el servicio o el producto tienen un alto grado de confiabilidad, el cliente lo hace suyo hasta llegar a personalizar el consumo.

Los estudios administrativos al respecto buscan entender $y$ anticiparse a las necesidades del cliente. Por ejemplo, les dan mayores ofertas, explotan sus potencialidades, les otorgan costos especiales y hasta les buscan nuevas perspectivas, haciéndolos sus clientes estrellas.

En la administración educativa, se ha subestimado a los clientes como padres de familias, colaboradores, alumnos estrellas e, incluso, empresas que facilitan y colaboran para que nuestras escuelas presten servicios excelentes. Algunos directores o promotores cuando han manejado esta forma de agradar a sus usuarios, lo han hecho atrayéndolos con becas o regalos especiales. De esta manera, han tomado más en cuenta una estrategia de negocio que un manejo del factor humano en la educación.

Esta vez, es necesario ver las cosas de otro modo. Se debe lograr que la confiabilidad se trasforme en un factor de acercamiento, de conocimiento y ayuda para mejorar la calidad educativa. Para ello, son necesarias las siguientes recomendaciones: 
a) Tener la información disponible de nuestros alumnos y padres de familia (es decir, de los miembros de las empresas con los cuales nos relacionamos) para mejorar el entendimiento y conocimiento del cliente.

b) Hacer visitas personales cuando los usuarios lo requieran, sin perder los valores de respeto, la amabilidad y la confianza. En muchos casos, es necesario hacerles ver que sentimos su ausencia.

c) Ser receptivos sobre sus opiniones, sus criterios y sus posibilidades; de modo que se sientan partícipes en la formación y desarrollo de la empresa educativa, aunque nosotros seamos los conductores.

d) Hacer sentir que la Dirección necesita de su apoyo y que cada uno de ellos es una singularidad.

e) Establecer un mérito preferencial cotidiano.

f) Mostrar la vocación de servicio a cada instante; sobre todo, cuando podemos contribuir a la solución de sus problemas educativos.

Para estos y otros datos de confiabilidad, que se hacen pero que pocas veces se resaltan, tendremos que ser muy proclives a lo siguiente:

a) Tener una base de datos sin muchas complicaciones, cuya información rápida facilite la solución de problemas.

b) La administración debe estar proclive a ser receptiva, hasta lista para el cambio.

c) No ver en el usuario un cliente de ganancia, de cobro, ni llegar a tener la pasión del cliente mayor (mayor cliente = mayor ganancia). Es necesario lograr que la confiabilidad determine el incremento de clientes.

d) Facilitar los cambios, haciéndolos con la confiabilidad que el usuario necesite para no trastocar su formación y sus pagos. 
e) Comprender que un buen usuario es un apoyo para la Dirección y la empresa educativa; por lo tanto, para el ambiente de familia.

Las consecuencias para muchos centros educativos han sido siempre muy rentables y han determinado una línea de trabajo que por años le ha dado confiabilidad. El ejemplo característico son los colegios en los que las familias confían la formación de sus hijos por varias generaciones. En las instituciones educativas, la formación determina instituciones emblemáticas de un sistema educativo. Se ha reconocido que cuando la atención al cliente falla, puede suceder lo siguiente:

a) Se produce la decadencia organizacional y la pérdida de alumnos.

b) Se tiene padres de familia poco colaboradores.

c) Existe la falta de datos que impiden planificar o mejorar la enseñanza en los colegios.

d) Existe dificultad para evaluar procesos.

e) Ocurren quejas frecuentes del cliente, quien realiza propaganda negativa y hasta logra denunciar sin bases legales.

Se debe tener en cuenta las maneras de lograr la eficiencia de la institución educativa, pero sin dejarse manejar por el cliente.

\section{ESTUDIO DE UNA MATRIZ PARA ESTABLECER UNA CONFIABILIDAD EDUCATIVA}

La matriz es una manera de captar las ideas en forma más objetiva y práctica. En este estudio, se trata de buscar y hacer algo que pueda ser 
valorativo o manejable. Es decir, se facilita la precisión del trabajo desde lo cualitativo hasta lo cuantitativo.

Dentro del trabajo educativo, las matrices son ejemplos que pueden alcanzar el grado de un nuevo manejo según la creatividad de aquello que deseamos evaluar o precisar.

\subsection{MATRIZ DE JERARQUÍAS}

El trabajo científico que desea buscar la confiabilidad necesita de una matriz que facilite la mayor comprensión del trabajo. El equipo planificador y ejecutor del proyecto educativo es quien realiza esta labor, teniendo en cuenta su experiencia y el conocimiento de lo que debe hacerse y de la institución a la cual determina el desempeño del trabajo educativo. En esa matriz, se establecen calificaciones estándares y posibilidades con frecuencias que vayan indicando lo que sucede o puede suceder.

La forma más simple de hacer una matriz es teniendo en cuenta los factores universales, que se mostrarán a continuación:

a) Establecer los factores y los subfactores: Quiere decir que la confiabilidad se determina en áreas (factores) cuyo valor determina las variables en las cuales se basa el trabajo. Junto a ello, están los aspectos de jerarquía (variables dependientes o subfactores).

b) Determinar los indicadores: Estos valores cuantitativos sirven para establecer si un factor o subfactor, de menor a mayor, determina con una numeración el grado de confiabilidad dentro de la eficiencia. 


\subsection{MATRIZ PARA PODER ESTABLECER LA CONFIABILIDAD EDUCATIVA}

Se debe establecer la matriz de confiabilidad teniendo en cuenta que los factores universales de confiabilidad son los más comunes; sin embargo, se pueden establecer otros según les convenga a las instituciones educativas. En ese caso, se puede tener en cuenta los aspectos económicos, políticos, educativos, entre otros. De igual manera, las jerarquías y los indicadores pueden modificarse según la clase de investigación que se propone el administrador educativo. Para primer caso, las jerarquías son productos de las necesidades a las cuales se recurren para esclarecer la confiabilidad; para el segundo, los indicadores son manifestaciones de un claro valor con el cual queremos precisar los detalles en números para transformarlos en porcentajes.

Para el caso que se va a tratar, se tendrán en cuenta los factores, los aspectos de las jerarquías (como si fueran las variables) y los indicadores. En los factores se indican todos aquellos que se necesitan para especular con características de criterios de la confiabilidad. Los aspectos de las jerarquías serán los detalles en los cuales los factores se desviaran para especular con mayor precisión lo que se desea establecer dentro de la confiabilidad. Los indicadores son precisiones cuantitativas o la objetividad para evaluar con mayor certeza. En todas estas formas de determinar los aspectos de la matriz, se pretende que el referente trate de buscar otras formas de hacer con el fin de que busque la evaluación de la confiabilidad.

A continuación, se presenta la matriz para determinar la confiabilidad de un centro educativo de nivel inicial, primario y secundario. 
CÉSAR S. CORTEZ MONDRAGÓN

\begin{tabular}{|c|c|c|c|c|c|}
\hline \multirow{2}{*}{$\begin{array}{c}\text { Factores } \\
\text { universales de } \\
\text { confiabilidad }\end{array}$} & \multirow{2}{*}{$\begin{array}{l}\text { Aspectos de las jerarquías } \\
\text { (sub factores) }\end{array}$} & \multicolumn{4}{|c|}{ Indicadores } \\
\hline & & 1 & 2 & 3 & 4 \\
\hline \multirow{5}{*}{ Equipo } & Edad óptima para realizar el trabajo & & & & \\
\hline & Conocimiento de los temas & & & & \\
\hline & Experiencia en el trabajo por realizar & & & & \\
\hline & Cantidad adecuada de elementos & & & & \\
\hline & Capacidad de compromiso & & & & \\
\hline \multirow{4}{*}{$\begin{array}{l}\text { Medio } \\
\text { ambiente }\end{array}$} & Favorable para hacer el trabajo & & & & \\
\hline & Cuenta con el material adecuado & & & & \\
\hline & Existe apreciación social & & & & \\
\hline & El usuario tiene confianza en el equipo & & & & \\
\hline \multirow{6}{*}{$\begin{array}{l}\text { Carga del } \\
\text { trabajo }\end{array}$} & Probabilidad del monto de trabajo & & & & \\
\hline & Tiempo adecuado para realizar & & & & \\
\hline & Etapas adecuadas del proceso & & & & \\
\hline & Madurez del equipo & & & & \\
\hline & Aceptación de logros & & & & \\
\hline & Aceptación de fallas & & & & \\
\hline \multirow{4}{*}{$\begin{array}{l}\text { Posibilidades } \\
\text { de fallas }\end{array}$} & Existen fallas potenciales & & & & \\
\hline & Existen fallas funcionales & & & & \\
\hline & Capacidad de corrección & & & & \\
\hline & Margen de errores & & & & \\
\hline \multirow{3}{*}{$\begin{array}{l}\text { Capacidad de } \\
\text { valores }\end{array}$} & Es ético y moral hacerlo & & & & \\
\hline & $\begin{array}{l}\text { Equipo con testimonio de } \\
\text { responsabilidad }\end{array}$ & & & & \\
\hline & Profesional que ayuda al usuario & & & & \\
\hline \multirow{3}{*}{$\begin{array}{l}\text { Uso de } \\
\text { pruebas de } \\
\text { medición }\end{array}$} & Confección de pruebas & & & & \\
\hline & Calificación de pruebas & & & & \\
\hline & Coordinación de experiencias & & & & \\
\hline
\end{tabular}

El paso siguiente sería analizar las calificaciones de cada factor $y$, luego, la totalidad de estos, para poder ver el mérito de la confiabilidad.

Según los resultados (cantidades) que brinda cada indicador, se establece la calificación, teniendo en cuenta los números y, a través ellos, los porcentajes en dicha calificación. 
Se debemos tener en cuenta que el indicador con N. ${ }^{\circ} 1$ representa el 25\% de la confiabilidad; el N. ${ }^{\circ} 2$, el $50 \%$ de la confiabilidad; el N. o 3 , el 75\%, y el N.o 4, el 100\%.

Se expone, a continuación, el ejemplo con el factor «equipo»:

\begin{tabular}{|c|c|c|c|c|c|}
\hline \multirow{2}{*}{$\begin{array}{c}\text { Factores universales } \\
\text { de confiabilidad }\end{array}$} & \multirow{2}{*}{$\begin{array}{c}\text { Aspectos de las jerarquías } \\
\text { (subfactores) }\end{array}$} & \multicolumn{4}{|c|}{ Indicadores } \\
\hline & & 1 & 2 & 3 & 4 \\
\hline \multirow{5}{*}{ Equipo } & Edad óptima para realizar el trabajo & & & $\mathrm{X}$ & \\
\hline & Conocimiento de los temas & & & & $\mathrm{X}$ \\
\hline & Experiencia en el trabajo por realizar & & & $\mathrm{X}$ & \\
\hline & Cantidad adecuada de elementos & & $\mathrm{X}$ & & \\
\hline & Capacidad de compromiso & & & & $\mathrm{X}$ \\
\hline Totales & & 0 & 2 & 6 & 8 \\
\hline
\end{tabular}

El valor total de los indicadores es 16 .

El valor óptimo sería 20 , que equivaldría al $100 \%$ por ser el mayor total de la numeración cuatro.

Si se quisiera saber el grado de confiabilidad del factor «equipo», se tendría la siguiente ecuación:

$$
\underline{16 \times 100}=80 \%
$$

20

La conclusión sería que el «equipo» presenta una probabilidad de confiabilidad del $80 \%$, lo cual es meritorio para emprender la tarea. De esta manera, se puede establecer cada uno de los valores que tienen los factores y determinar el análisis de confiabilidad; así también se puede establecer el conjunto de todos los factores de la matriz. A continuación, se observará en un cuadro la síntesis del grado de aceptación. 
ACEPTADO CON ÉXITO

\begin{tabular}{|l|l|}
\hline $\begin{array}{l}\text { Decisión correcta } \\
\text { de confiabilidad: Desde } \\
\text { el 100\% hasta el } 90 \% .\end{array}$ & $\begin{array}{l}\text { Decisión no confiable: } \\
\text { Desde el } 60 \% \text { hasta el } 50 \% .\end{array}$ \\
\hline $\begin{array}{l}\text { Confiabilidad con error de } \\
\text { aceptación: }\end{array}$ & $\begin{array}{l}\text { Rechazo en la confiabilidad: } \\
\text { Desde el } 80 \% \text { hasta el } 70 \% .\end{array}$ \\
\hline
\end{tabular}

\section{EL CONTROL COMO PARTE DE LA CONFIABILIDAD EN LAS TAREAS EDUCATIVAS: ACADÉMICAS Y ADMINISTRATIVAS}

Como su nombre lo indica, el control es una forma de seguir el cumplimiento de los objetivos y de las metas dentro de una empresa o institución educativa. Es decir, nada debe ser confiable, sino donde se demuestra que aquello que se hace, se está haciendo bien.

Dentro de la confiabilidad, los controles confirman y no tienen el carácter ni de ser coactivos ni determinantes para producir un cambio; sin embargo, ayudan para hacer que la confiabilidad se mueva con mayor precisión. Por lo tanto, la confiabilidad se vuelve evidente mediante el uso del control.

En verdad, para los educadores, la confianza se encuentra en el grado de control permanente; por eso, se establecen las formas de ver cómo se va desarrollando desde una actividad simple hasta un plan estratégico o un plan operativo dentro de los tiempos que se han determinado.

De los anteriores conceptos, se desprenden formas y necesidades de control en un plan o adjunto al desarrollo de actividades y participación de elementos que ejecutan esos sistemas de control.

Sin pretender imponer formas de control, porque se quitaría la creatividad y la eficacia al manejar la evaluación de las instituciones, 
los controles, dentro de la confiabilidad, pueden darse con las siguientes características:

a) Como parte del sistema interno educativo y con estructuras dentro de los planes estratégicos u operativos. Algunas empresas, por su envergadura y amplitud, lo trabajan en un aparte. Eso quiere decir que es una estructura complementaria.

b) Debido a la dinámica educativa, la creatividad de los profesores, padres de familia, directivos y hasta las proyecciones del grupo social al que califica, la confiabilidad determina que los controles valoran. Por lo tanto, en muchos casos pueden quedar cortos y es necesario reajustarlos o ampliarlos.

c) Para que el control no se agobiante, debe estar comprendido con actividades o tareas ordinarias; por ejemplo, las pruebas simples, las calificaciones ordinarias, las encuestas de fin de ciclo, etc., de modo que se pueda ir formando una cultura de evaluación dentro de la institución.

d) En todo lo que se relaciona con la educación en el actuar humano, se parte del proceso de mejorar el sistema y de contribuir a mejorar la calidad de la educación. La confiabilidad contribuye a reafirmarlos. para prevenir males mayores.

e) El control de trabajo interno, como la confiabilidad, está en razón directa de la complejidad de la institución. Cuando es más compleja la escuela o colegio, mayor será el sistema de control. En algunos casos, son simples evaluaciones de proceso; mientras que en otros casos, cuando tienen alcance nacional e internacional, serán sistemas estructurales independientes al manejo cotidiano de la institución. 


\subsection{TÉCNICAS Y FORMAS MÁS FRECUENTES DE CONTROL}

En el presente trabajo, no se pretende explicar cada una de las técnicas de control que se puedan dar en un sistema educativo o en una administración educativa, menos para determinar la validez del aprendizaje. Muchos de ellos son muy conocidos por los educadores. Lo que se pretende es hacer notar son todas aquellas formas que, como técnicas de control, sirven para hacer más confiable el servicio educativo.

a) Sistemas de información: Son todos aquellos datos que sirven de referencia para precisar la confiabilidad. Ayudan en cuanto a sus índices cuantitativos y confirman que no habrán fallas ni riesgos. Estos son los siguientes:

—Datos de contabilidad

—Datos de auditoria

—Informes de presupuestos

-Reportes o informes sobre casos

- Sistemas o programas computarizados (base de datos)

b) Gráficas: Son las representaciones que señalan, en forma integrada, los datos. Su objetividad permite dar confiabilidad al trabajo. Para que sirvan de ayuda, se debe tener presente lo siguiente:

—Claridad y sencillez en la interpretación

-Capacidad para indicar el proceso que explica

-Uso frecuente en el manejo

—Objetividad que denote fácil uso 
c) Estudio de métodos: Algunos educadores basan su confiabilidad en el uso de métodos, sobre todo cuantitativos. Siempre se recomienda que sirvan de índice de confirmación de otros datos. Si existe una variación, es necesario complementar con informes que ayuden a ver la exactitud, la determinación, el cumplimiento de las normas de calidad, etc. Entre estos métodos de estudio, se tienen los siguientes:

—Controles de tiempos y movimientos

—Estudios de estándares

-Modelos matemáticos referenciales

—Explicación de redes como caminos críticos (PERT)

—Cálculos de probabilidad

—Resultados de programas dinámicos

\section{Conclusiones}

8. Un sistema educativo es confiable cuando no hay fallas, los riesgos son mínimos y los errores son controlables.

9. En educación, una persona es confiables cuando realiza su trabajo con eficiencia y eficacia, tiene iniciativa y creatividad, mejora sus fallas y trata de convertir sus errores en virtudes y eficiencias. Los problemas son experiencias que ayudan a buscar soluciones.

10. Una institución educativa es confiables cuando llena las expectativas de sus usuarios, se les ayuda a conseguir sus propósitos y se les favorece en su desarrollo y en el crecimiento que se han propuesto. 
11. Una clase es confiable cuando se sabe que los educandos aprenden sin coacción y con eficiencia, cuando desean estudiar y cuando disfrutan de su estadía. Por ello, el profesor se siente satisfecho de su labor y llega a alcanzar progresos insospechables; además, realza la labor profesional con todo mérito y dedicación. De igual manera, el padre de familia nota el adelanto en la formación de sus hijos (académico, técnico y valorativo) y, sobre todo, en el crecimiento como persona dentro de la sociedad.

12. Una tarea educativa es confiable cuando se observa que todo está planificado. De esta forma, se siente el orden, pues cada miembro está en el lugar donde tiene que ejecutarse la tarea. Así, cuando se presenta algún imprevisto, la colaboración sobra, porque se transforma en algo leve.

13. Una prueba de evaluación educativa es confiable cuando, probada en varias formas y alternativas, con diferentes muestras, sus resultados son parecidos; por tanto, ayuda a confirmar aquello que se quiere mejorar. Sirve de base para los actos superables y es el diagnóstico posible de una solución.

14. La confiabilidad no se da por decretos, ni se establece por mandatos.

15. Los tiempos son tiempos de mucha técnica; en estos, se establece la confiabilidad.

16. En educación, la confiabilidad es el valor que está más allá de la gestión del aprendizaje y de la enseñanza, de realizar todo acto educativo dentro de un sistema para la formación de las personas.

17. Por último, la confiabilidad no es eterna, porque no son eternos ni estáticos nuestros aprendizajes ni la capacidad de formación dentro de la sociedad en que nos situamos. Mucho depende de los valores humanos adquiridos y practicados, de las posibles circunstancias a 
las que somete la experiencia cotidiana, de los elementos técnicos que se usan para realizar el bien de otras personas; pero, sobre todo, estará sujeto — como dijo un gran educador y maestro- a la dosis de amor que se ponga en el acto educativo y al mérito del trabajo que se realiza para hacer el bien al prójimo. 


\section{BIBLIOGRAFÍA}

Carrasco Díaz, Sergio

2006 Metodología de la investigación cientifica. Lima: Editorial San Marcos.

Dessler, Gary

1998 Administración de personal. México D. F.: Hispanoamericana S.A.

ELwood, S. Buffon

1982 Administración y dirección técnica de la producción. México D. F.: Limusa.

KRICK, Edward V.

1961 Ingeniería de métodos. México D. F.: Editorial Limusa.

Manrique Enríquez, Fernando

2002 Teoría de los valores y ética. Lima: Colección Exísteme.

Oficina Internacional del Trabajo (OIT)

2001 Introducción al estudio del trabajo. 4. ${ }^{a}$ Edición. México D. F.: Editorial Limusa.

Planeta Ediciones

2000 Diccionario de ciencia y tecnología. Madrid: Planeta Ediciones. 
Salkind, Neil

1999 Metodología de la investigación. México D. F.: Editorial Printice Hall. Universidad de Kansas.

ROBBINS, Stephen P.

1997 La administración en el mundo de hoy. México D. F.: Prentice Hall.

SExto, Luis Felipe

s. f. El ciclo de calidad: qué tontería más genial. <http://www. confiabilidad.net.>

Sherman, Arthur; George Bohlander y Scout Snel

1999 Administración de recursos humanos. México D. F.: Thomas Editores.

Tomerz Arroyo, Fernando

2003 Administración de confiabilidad de los procesos. I Congreso Mexicano en Contabilidad y Mantenimiento. 